\title{
Using artificial intelligence for effective decision-making in corporate governance under conditions of deep uncertainty
}

\author{
Aleksandra Volosova ${ }^{1, *}$, and Ekaterina Matiukhina ${ }^{2}$ \\ ${ }^{1}$ Faculty of Management, Automated control systems, Automobile and Road Construction State \\ Technical University (MADI), Leningradsky prospect, 64, 125319 Moscow, Russia \\ ${ }^{2}$ Russian Technological University (MIREA), Vernadsky Avenue, 78, 119454 Moscow, Russia
}

\begin{abstract}
The article deals with research related to the use of artificial intelligence technologies for effective decision-making in corporate governance under conditions of deep uncertainty. To process uncertainty, it is proposed to use the cognitive capabilities of artificial intelligence. Cognitivism can be used to implement intuitive, psychological and other components of the internal mental activity of a person when making decisions. These capabilities allow one to make informed decisions and predict the consequences of these decisions. To study the properties of deep uncertainty, the authors suggest using a tensor model. The tensor model of deep uncertainty makes it possible to study additional properties of uncertainty that are not available in traditional models, such as Bayesian formalism, Dempster-Shafer theory, fuzzy sets, a method based on certain factors (Stanford formalism), and others. The use of the tensor model allows one to study the spatial model of uncertainty, real and imaginary values of uncertainty, as well as uncertainty invariants with respect to various transformations of the coordinate system.
\end{abstract}

\section{Introduction}

Technological transformation and coronavirus has had a serious impact on the concept of management. To improve the efficiency of corporate governance, it is necessary to take into account new business formats. Corporate management of ecosystem, platform, and distributed companies is a highly computationally complex task. Artificial intelligence technologies are now being successfully used to solve such problems.

Tasks such as:

- data collection and analysis

- monitoring the organization's own activities

- monitoring the workforce problems

- representation of the company's brands, products and services in social networks, monitoring user views of this information

- $\quad$ risk management procedures

\footnotetext{
*Corresponding author: avolosova32@gmail.com
} 
- market forecasts

- analysis of existing and forecasting new socio-cultural trends to monitor their impact on business

- monitoring the company's position on various social issues

- forming and optimizing the organization's own rating and analyzing the impact of the rating on the stock market, investors, consumers, and social networks.

- forecast the organization's investment in various areas in order to avoid the financial collapse of the organization

- assessment of risk factors

- data collection and analysis management decision-making, personnel decisionmaking, internal and external financial policy decision-making, disaster decisionmaking can be solved using artificial intelligence.

Now the penetration of AI into the daily lives of people and businesses is undeniable [3]. Obviously, artificial intelligence also has an impact on corporate governance. The complexity and rapid change of the world make making the right decisions a very difficult process [5][6][7][11]. And often the cost of mistakes is very high [1]. Of course, you can use automation to partially reduce risks. But it is better to use the cognitive capabilities of artificial intelligence. The cognitive capabilities of artificial intelligence can be used to implement intuitive, psychological and other components of the internal mental activity of a person when making decisions. The cognitive capabilities of artificial intelligence allow one to make informed decisions and predict the consequences of these decisions. Usually, the information that a person uses to make decisions contains noise that changes the measured values [2]. So we are dealing with uncertainty. And if decisions are made in dynamic interaction with the system, then there is such a thing as deep uncertainty. Decision-making under deep uncertainty is a complex process that which combines artificial intelligence technologies such as fuzzy knowledge processing and forecasting. Next, we consider possible approaches to handling deep uncertainty in the decision-making process. When we try to represent uncertainty in the knowledge base, we may encounter the following problems:

- the degree of uncertainty of information is so high that during formalization, an erroneous representation of this information inevitably occurs

- based on poorly presented knowledge, erroneous conclusions may be drawn.

In other words, the success of making a decision depends on how we present the uncertainty.

When using traditional tools for mathematical modeling of uncertainty, such as Bayesian formalism, Dempster-Shafer theory, fuzzy sets, the method based on certainty factors (Stanford formalism) and their various combinations and modifications, we cannot say that the resulting model fully reflects all the properties of uncertainty. This is due to the unavoidable use of heuristics in modeling uncertainty. We can't avoid using heuristics to formalize uncertainty, but we can try to minimize its impact on our research. To do this, consider the tensor basis and try to represent the uncertainty using this basis.

\section{Tensor method of dual networks}

The fuzzy set basis can be used to handle uncertainty [4]. To formalize uncertainty we use the a fuzzy quantity. A fuzzy quantity can be defined as an arbitrary fuzzy set:

$$
\mathrm{Z}=\left\{\zeta, \vartheta_{\mathrm{Z}}(\zeta)\right\}, \zeta \in \mathrm{Z}
$$


This set $\zeta$ defined on the set of real numbers $\mathrm{R}$ and the membership function of a fuzzy quantity is a function of the form:

$$
\vartheta_{\mathrm{Z}}(\zeta) \rightarrow R[0,1]
$$

Define $\mathfrak{I}$ as tensor:

$$
\begin{gathered}
\mathfrak{I}=\zeta \otimes\left(\vartheta_{\mathrm{Z}}(\zeta)\right)^{T}(3) \\
\mathfrak{I}=\left\{\zeta_{1}, \zeta_{2} \ldots \zeta_{n}\right\} \otimes\left\{\begin{array}{c}
\vartheta_{\mathrm{Z}}\left(\zeta_{1}\right) \\
\vartheta_{\mathrm{Z}}\left(\zeta_{2}\right) \\
\ldots \\
\vartheta_{\mathrm{Z}}\left(\zeta_{n}\right)
\end{array}\right\}=\left\{\begin{array}{cccc}
\zeta_{1} * \vartheta_{\mathrm{Z}}\left(\zeta_{1}\right) & \zeta_{2} * \vartheta_{\mathrm{Z}}\left(\zeta_{1}\right) & \ldots & \zeta_{n} * \vartheta_{\mathrm{Z}}\left(\zeta_{1}\right) \\
\zeta_{1} * \vartheta_{\mathrm{Z}}\left(\zeta_{2}\right) & \zeta_{2} * \vartheta_{\mathrm{Z}}\left(\zeta_{2}\right) & \ldots & \zeta_{n} * \vartheta_{\mathrm{Z}}\left(\zeta_{2}\right) \\
\ldots & \ldots & \ldots & \ldots \\
\zeta_{1} * \vartheta_{\mathrm{Z}}\left(\zeta_{n}\right) & \zeta_{2} * \vartheta_{\mathrm{Z}}\left(\zeta_{n}\right) & \ldots & \zeta_{n} * \vartheta_{\mathrm{Z}}\left(\zeta_{n}\right)
\end{array}\right\}
\end{gathered}
$$

So a fuzzy variable can be represented by a tensor.

Using the transformation, we can obtain from the dyad a symmetric tensor, an antisymmetric tensor, a sphere tensor, and a deviator. And we can determine additional properties of the fuzzy variable as a result of analyzing these tensors.

\section{Results of applying the tensor basis for the system modeling of deep uncertainty}

Let's consider the application of the tensor basis for modeling uncertainty in the problem of forecasting passenger traffic volumes in St. Petersburg and the Leningrad region for the period up to $2030[8][9][10][12]$. The calculation is made for conservative and innovative options (See Tab.1, Tab. 2).

Table 1. Passenger traffic trends to 2030 (conservative option)

\begin{tabular}{|l|l|l|l|}
\hline & $\mathbf{2 0 2 0}$ & $\mathbf{2 0 2 5}$ & $\mathbf{2 0 3 0}$ \\
\hline Passenger traffic (million people) & $\mathbf{2 0 6 8 , 9}$ & $\mathbf{2 1 3 0}$ & $\mathbf{2 1 9 4 , 9}$ \\
\hline Saint-Petersburg & 1971,3 & 2027 & 2086,9 \\
\hline Leningrad region & 97,6 & 103 & 108 \\
\hline
\end{tabular}

Table 2. Passenger traffic trends to 2030 (innovative option)

\begin{tabular}{|l|l|l|l|}
\hline & $\mathbf{2 0 2 0}$ & $\mathbf{2 0 2 5}$ & $\mathbf{2 0 3 0}$ \\
\hline Passenger traffic (million people) & $\mathbf{2 1 3 9 , 1}$ & $\mathbf{2 2 1 6 , 9}$ & $\mathbf{2 3 2 2 , 3}$ \\
\hline Saint-Petersburg & 2040,7 & 2108,4 & 2202,4 \\
\hline Leningrad region & 98,4 & 108,5 & 119,9 \\
\hline
\end{tabular}

Passenger traffic for each year is a fuzzy value. Hence the following fuzzy variables can be defined for each calculation option (conservative and innovative) (See Tab. 3). 
Table. 3. Fuzzy variables

\begin{tabular}{|l|l|}
\hline Conservative option & Innovative option \\
\hline & $\zeta_{1 i}-$ fuzzy $2139.1(\mathbf{2 0 2 0})$ \\
$\zeta_{1 c}-$ fuzzy $2068.9(\mathbf{2 0 2 0})$ & $\zeta_{2 i}-$ fuzzy $2216.9(\mathbf{2 0 2 5})$ \\
$\zeta_{2 c}-$ fuzzy $2130(\mathbf{2 0 2 5})$ & $\zeta_{3 i}-$ fuzzy $2322.3(\mathbf{2 0 3 0})$ \\
$\zeta_{3 c}-$ fuzzy $2194.9(\mathbf{2 0 3 0})$ & \\
\hline
\end{tabular}

Now we define universums and membership functions for fuzzy variables (See Tab. 4).

Table 4. Universums and membership functions for fuzzy variables

\begin{tabular}{|c|c|c|}
\hline & conservative option & innovative option \\
\hline 2020 & $\begin{array}{l}\zeta_{1 c}=[1906: 26.84: 2100] \\
\vartheta_{Z}\left(\zeta_{1 c}\right)=\operatorname{trimf}\left(\zeta_{1 c},[1907.862068 .92081 .12]\right)\end{array}$ & $\begin{array}{l}\zeta_{1 i}=[1852.94: 40.88: 2220.86] \\
\vartheta_{Z}\left(\zeta_{1 i}\right)=\operatorname{trimf}\left(\zeta_{1 i},[1893.822139 .12179 .98]\right)\end{array}$ \\
\hline 2025 & $\begin{array}{l}\zeta_{2 c}=[2044.46: 12.22: 2154.44] \\
\vartheta_{Z}\left(\zeta_{2 c}\right)=\operatorname{trimf}\left(\zeta_{2 c},[2056.6821302142 .22]\right)\end{array}$ & $\begin{array}{l}\zeta_{2 i}=[2108.04: 12.98: 2248.86] \\
\vartheta_{Z}\left(\zeta_{2 i}\right)=\operatorname{trimf}\left(\zeta_{2 i},[2123.542216 .92232 .46]\right)\end{array}$ \\
\hline 2030 & $\begin{array}{l}\zeta_{3 c}=[2114.04: 12.98: 2220.86] \\
\vartheta_{Z}\left(\zeta_{3 c}\right)=\operatorname{trimf}\left(\zeta_{3 c},[2127.022194 .92207 .88]\right)\end{array}$ & $\begin{array}{l}\zeta_{3 i}=[2174.74: 21.08: 2364.46] \\
\vartheta_{Z}\left(\zeta_{3 i}\right)=\operatorname{trimf}\left(\zeta_{3 i},[2195.822322 .32343 .38]\right)\end{array}$ \\
\hline
\end{tabular}

The Fig.1 (a-c) shows the fuzzy variables for the conservative and innovative options. So we can perform all the operations available to us from fuzzy set theory on variables. But each variable has a quantity behind it. In our case, this is passenger traffic. By studying the fuzzy set, we can draw conclusions about the fluctuations of this quantity within the boundaries of the fuzzy set. However, we can't say anything about this quantity beyond these boundaries. This limitation can be overcome by using a tensor basis. Applying formula 3 to the vector of values of each fuzzy variable and its membership function, we obtain tensors of fuzzy variables (see Fig. $1 \mathrm{~d}-\mathrm{j}$ ). 

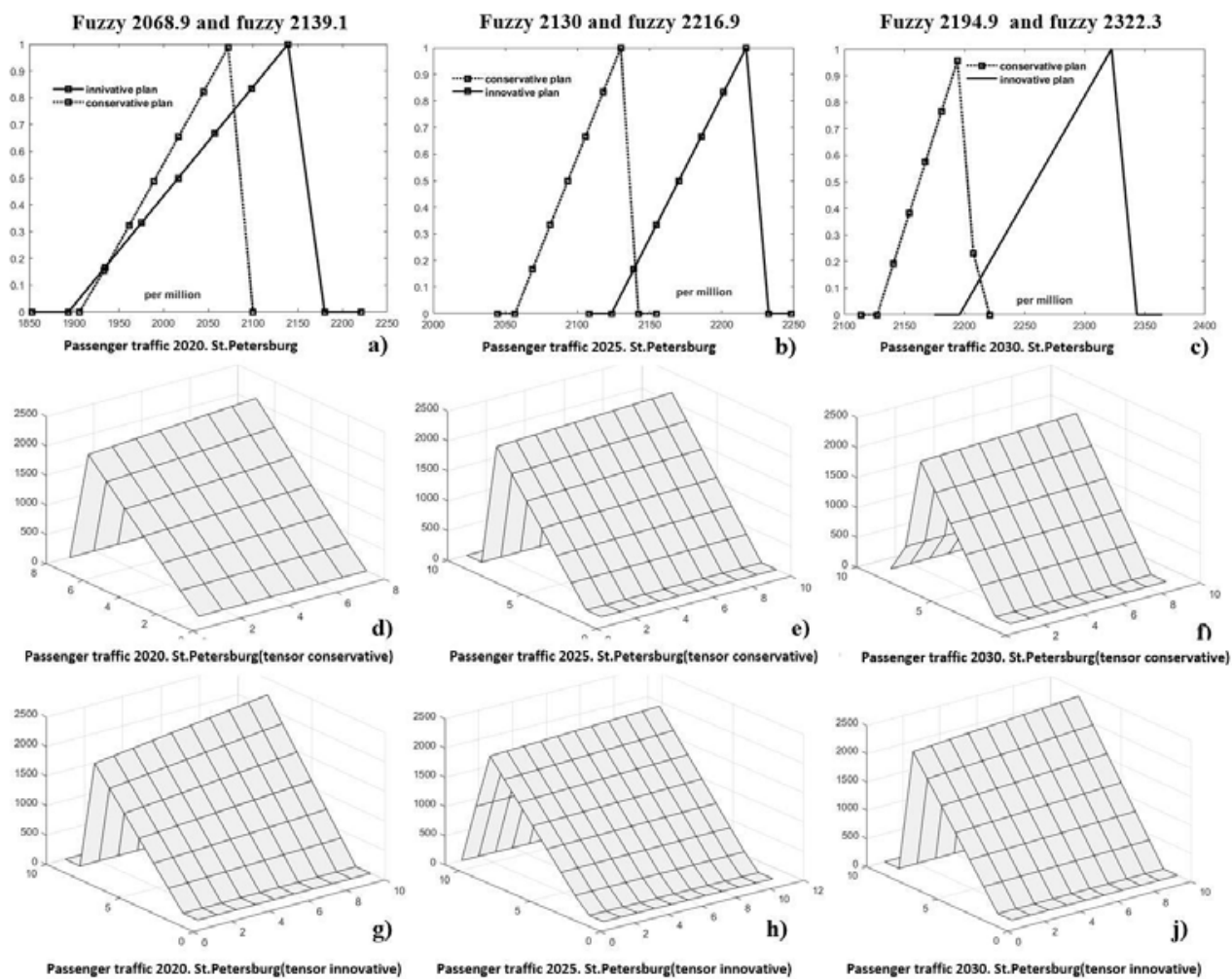

Fig. 1. The fuzzy sets and tensors

Hence instead of a set of values for a fuzzy variable we get a mathematical object and can now explore additional properties of uncertainty. Using the tools of tensor algebra, we can obtain additional tensors for each tensor of a fuzzy variable. As an example, we decompose the tensor for fuzzy 2068.9 into a sphere tensor and a deviator. The resulting tensors describe additional properties of this fuzzy variable - it's clear and fuzzy parts (see Fig. 2).

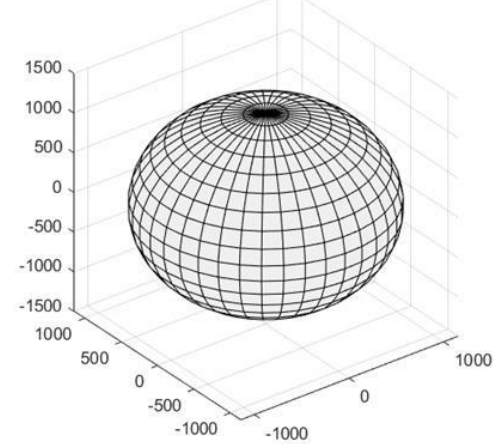

Sphere tensor for fuzzy $\mathbf{2 0 6 8 . 9}$

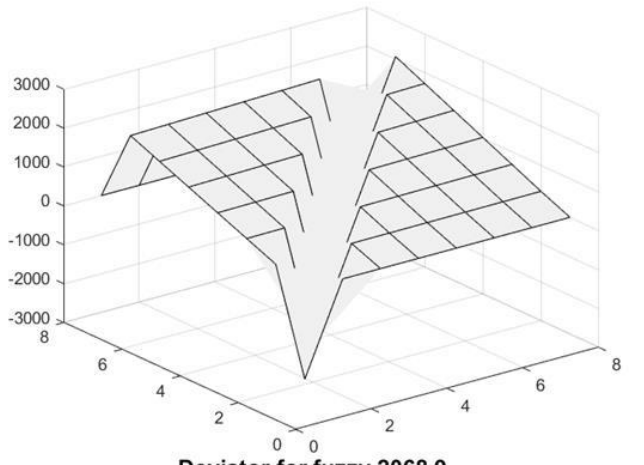

Deviator for fuzzy 2068.9

Fig. 2. The sphere tensor and deviator for fuzzy 2068.9

We can also decompose the tensor for fuzzy 2068.9 into symmetric and antisymmetric tensors (see Fig. 3). 

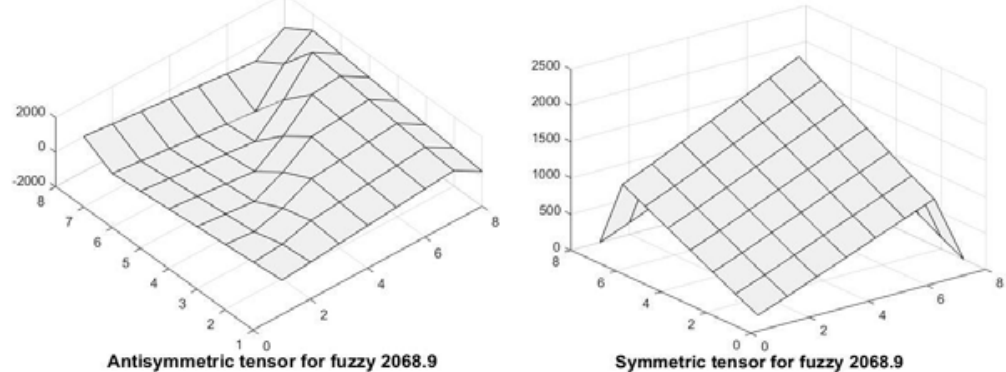

Fig. 3. The antisymmetric and symmetric tensors for fuzzy 2068.9

An antisymmetric tensor characterizes the transformation of a fuzzy tensor with respect to the base coordinate system. The antisymmetric tensor does not change when the coordinate axes are reflected and is constant in all coordinate systems where Newton's laws apply.

Finally, we can get the eigenvalues of the fuzzy variable tensor. The combination of these values forms the spectrum of the fuzzy variable tensor (See Fig. 4).
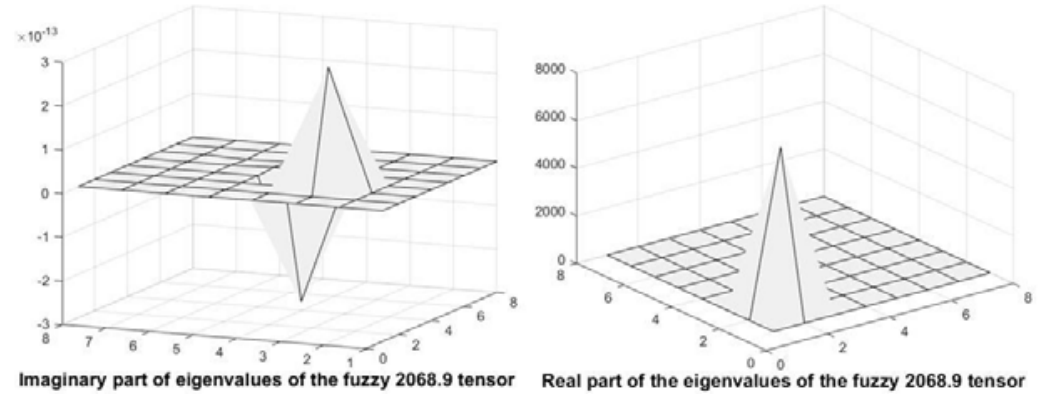

Fig. 4. The real and imaginary parts of the eigenvalues of the fuzzy 2068 tensor

So we see that the use of the tensor basis significantly expands the possibilities of studying the deep uncertainty that is an inevitable companion of artificial intelligence technologies. Especially when these technologies are used to solve complex corporate governance problems.

\section{Conclusions}

The research conducted in the article allows us to draw the following conclusions:

1. To improve the efficiency of corporate governance, it is necessary to take into account new business formats. This means using artificial intelligence technologies

2. The cognitive capabilities of artificial intelligence can be used to implement intuitive, psychological and other components of the internal mental activity of a person when making decisions. These capabilities allow one to make informed decisions and predict the consequences of these decisions.

3. Effective decisions in the corporate governance process are made under conditions of deep uncertainty, as these decisions are made in dynamic interaction with the management system

4. The tensor model of deep uncertainty allows you to explore additional properties of uncertainty that are not available when using traditional models, such as Bayesian 
formalism, Dempster-Shafer theory, fuzzy sets, and a method based on certain factors (Stanford formalism), etc. The tensor model is a spatial model of uncertainty; its real and imaginary values and invariants with respect to various transformations of the coordinate system.

\section{References}

1. O.I. Maksimychev, V.B. Golubkova, V.A. Vinogradov, Zav Zav Aung, The Integration of a Set of Functions for the Product Life Cycle of a Virtual Enterprise, Advances in Business and Finance, 2018 3rd BEM International Conference on Education, Sociology and Humanities (BEM-ESH 2018). August 14-16 (2018).

2. Kochenderfer, Mykel J., Decision making under urcentainty: theory and application (1980)

3. B.S. Subbotin and E.U. Ahmetzhanova, Research on the integration of intelligent transport systems, IOP Conference Series: Materials Science and Engineering, 832 (2019).

4. A.V. Volosova, Computational nanotechnology,1, (2019)

5. D. Akimov, P. Krug, A. Ostroukh, V. Ivchenko, T. Morozova, I. Sadykov, International Journal of Applied Engineering Research, 11(9), 6435 (2016)

6. E.Matyukhina, A. Ignatov, V. Ivchenko, P. Krug, T. Morozova, Applied Mechanics and Materials, 851, 470 (2016)

7. L.V.Zhivoglyadova, V.G. Zhivoglyadov and V.V. Siliyanov, IOP Conference Series: Materials Science and Engineering, International Conference on Digital Solutions for Automotive Industry, Roadway Maintenance and Traffic Control (DS ART 2019) 1 November 2019, Cholpon-Ata, Kyrgyzstan, 832 (2019)

8. D.B. Efimenko, V.M. Vlasov, V.N. Bogumil, V.A. Demin and S. A. Filatov, IOP Conference Series: Materials Science and Engineering, International Conference on Digital Solutions for Automotive Industry, Roadway Maintenance and Traffic Control (DS ART 2019) 1 November 2019, Cholpon-Ata, Kyrgyzstan, 832 (2019)

9. N. Novikov, S.V. Eremin, A.G. Shevtsova, Development of a methodology for optimizing the urban route in the metropolitan area, IOP Conference Series: Materials Science and Engineering, International Conference on Digital Solutions for Automotive Industry, Roadway Maintenance and Traffic Control (DS ART 2019) 1 November 2019, Cholpon-Ata, Kyrgyzstan, 832 (2019)

10. E. Matyukhina, A. Ignatov, V. Ivchenko, P. Krug, S. Pavelyev, Applied Mechanics and Materials, 851, 477 (2016)

11. E. Matyukhina, D. Akimov, A. Ostroukh, V. Ivchenko, P. Krug, ARPN Journal of Engineering and Applied Science, 12(11), 3433 (2017)

12. A comprehensive plan of transport services for the population of St. Petersburg and the Leningrad Region for the medium and long term (until 2030) in terms of suburban passenger transportation, approved by the order of the Government of the Leningrad Region dated 26.06.2019, 592 (2019) 